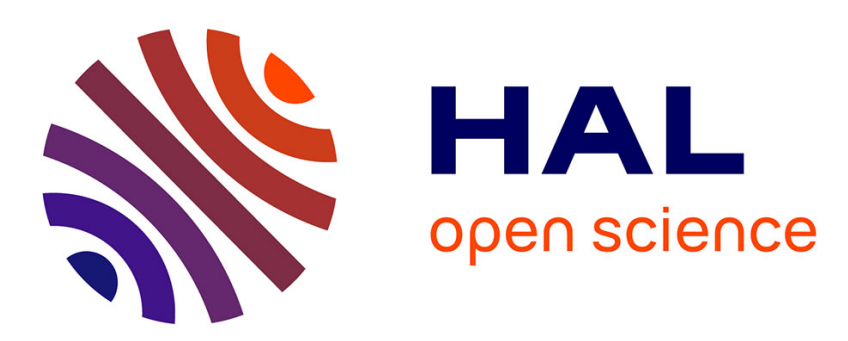

\title{
Importance des modalités d'imagerie dans la reproduction de configurations corporelles simples
}

Nicolas Robin, Lucette Toussaint, Yannick Blandin

\section{To cite this version:}

Nicolas Robin, Lucette Toussaint, Yannick Blandin. Importance des modalités d'imagerie dans la reproduction de configurations corporelles simples. Science \& motricité: Revue scientifique de l'Association des Chercheurs en Activités Physiques et Sportives, 2006, pp.67 - 78. 10.3917/sm.057.78 . hal-01529490

\section{HAL Id: hal-01529490 \\ https://hal.science/hal-01529490}

Submitted on 30 May 2017

HAL is a multi-disciplinary open access archive for the deposit and dissemination of scientific research documents, whether they are published or not. The documents may come from teaching and research institutions in France or abroad, or from public or private research centers.
L'archive ouverte pluridisciplinaire HAL, est destinée au dépôt et à la diffusion de documents scientifiques de niveau recherche, publiés ou non, émanant des établissements d'enseignement et de recherche français ou étrangers, des laboratoires publics ou privés. 


\title{
IMPORTANCE DES MODALITÉS D'IMAGERIE DANS LA REPRODUCTION DE CONFIGURATIONS CORPORELLES SIMPLES
}

\author{
Nicolas Robin et al.
}

EDP Sciences | Movement \& Sport Sciences

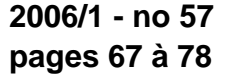

ISSN 2118-5735

Article disponible en ligne à l'adresse:

http://www.cairn.info/revue-science-et-motricite-2006-1-page-67.htm

Pour citer cet article :

Robin Nicolas et al., « Importance des modalités d'imagerie dans la reproduction de configurations corporelles simples ",

Movement \& Sport Sciences, 2006/1 no 57, p. 67-78. DOI : 10.3917/sm.057.78

Distribution électronique Cairn.info pour EDP Sciences.

(c) EDP Sciences. Tous droits réservés pour tous pays.

La reproduction ou représentation de cet article, notamment par photocopie, n'est autorisée que dans les limites des conditions générales d'utilisation du site ou, le cas échéant, des conditions générales de la licence souscrite par votre établissement. Toute autre reproduction ou représentation, en tout ou partie, sous quelque forme et de quelque manière que ce soit, est interdite sauf accord préalable et écrit de l'éditeur, en dehors des cas prévus par la législation en vigueur en France. II est précisé que son stockage dans une base de données est également interdit. 


\title{
Importance des modalités d'imagerie dans la reproduction de configurations corporelles simples
}

\author{
Nicolas Robin, Lucette Toussaint et Yannick Blandin ${ }^{(1)}$
}

\section{RÉSUMÉ}

Cette étude porte sur l'influence des modalités d'imagerie visuelle et proprioceptive sollicitées lors d'une pratique mentale sur le développement de la précision de la reproduction de configurations corporelles. Dans un premier temps (pré-test), 20 sujets ont été évalués sur leurs capacités à reproduire avec précision, sans l'aide de la vision, une position angulaire mobilisant l'articulation du genou. Ils ont ensuite été assignés aléatoirement soit à un groupe de pratique par imagerie visuelle, soit à un groupe de pratique par imagerie proprioceptive (90 essais de pratique). Des post-tests ont été réalisés 10 minutes et 24 heures après cette phase de pratique. Les résultats obtenus montrent que l'imagerie proprioceptive permet aux sujets de réduire leurs erreurs de positionnement angulaire. À l'opposé, une pratique sollicitant l'imagerie visuelle s'accompagne d'une détérioration temporaire de la performance. Ces résultats suggèrent la nécessité de conserver à l'identique la modalité dominante de la tâche à apprendre et la modalité d'imagerie sollicitée lors d'une pratique mentale.

Mots-clés : configurations corporelles, contrôle moteur, modalités d'imagerie, pratique mentale.

(1) Laboratoire d'Analyse de la Performance Motrice Humaine (LAPMH), MSHS, 99 avenue du Recteur Pineau, 86000 Poitiers, France. Correspondance : Lucette Toussaint, LAPMH, MSHS, 99 avenue du Recteur Pineau, 86000 Poitiers - Tél. : 0549454698 - Fax : 0549454657 lucette.toussaint@mshs.univ-poitiers.fr. 


\title{
Importance of imagery modalities when reproducing simple body configurations
}

\begin{abstract}
The influence of visual imagery and proprioceptive imagery in mental practice was investigated on the development of accurate body configurations reproduction. Twenty participants were first evaluated on their abilities to accurately produce body configurations without vision (pre-test). They were then randomly assigned to a visual or to a proprioceptive imagery practice group (90 trials of practice). Two post-tests were performed 10 minutes and 24 hours later. Results showed that proprioceptive imagery was more effective than visual imagery when learning to accurately reproduce knee joint angle. Moreover, visual imagery practice induced temporary performance decrement. These results suggest the necessity to maintain constant the dominant sensory modality of the task and the imagery modality used in mental practice.
\end{abstract}

Key words: body configurations, imagery modalities, mental practice, motor control.

\section{Introduction}

L'imagerie mentale est un processus conscient durant lequel les sujets simulent intérieurement une action motrice sans la réaliser réellement. Il est bien connu que cette pratique s'accompagne d'une amélioration de la performance, bien que celle-ci soit moindre comparée à une pratique physique réelle (Decety, Jeannerod, Germain et Pastene, 1991 ; Feltz et Landers, 1983 ; Hall, Buckolz et Fishburne, 1992). L'amélioration de la performance motrice suite à une pratique par imagerie ou à une pratique physique réelle pourrait attester de leur similarité fonctionnelle ou du partage d'un système commun de représentation des actions. Cette hypothèse est validée par plusieurs travaux reposant sur des paradigmes expérimentaux variés. L'utilisation d'un paradigme de chronométrie mentale a permis d'observer la similitude des aspects temporels d'actions imagées ou réellement produites (Decety, Jeannerod et Prablanc, 1989; Decety et Michel, 1989). Cette similitude des effets liés à une pratique réelle ou imagée ressort également de l'étude des caractéristiques spatiotemporelles des activités motrices, telles que décrites par la loi de Fitts (Decety et Jeannerod, 1996), de mesures d'indices physiologiques comme les rythmes cardiaque et respiratoire (Decety, Jeannerod, Durozard et Baverel, 1993), les réponses du système nerveux végétatif (Roure, Collet, Deschaumes-Molinaro, Delhomme, Dittmar et Vernet-Maury, 1999) et 
les tracés électromyographiques (Feltz et Landers, 1983). Les méthodes d'imagerie cérébrale fonctionnelle (TEP, IRMf) ont permis, quant à elles, de déterminer l'implication de structures cérébrales communes à ces deux types de pratique (Ingvar \& Philipsson, 1977 ; Jeannerod, 1999 ; Sirigu, Duhamel, Cohen, Pillon, Dubois et Agid, 1996).

Certains auteurs se sont intéressés plus particulièrement à la nature des images mentales sollicitées pour une efficacité maximale de l'apprentissage. Les consignes données aux sujets peuvent les guider vers des stratégies d'imagerie mentale sollicitant la modalité visuelle ou la modalité proprioceptive. L'imagerie visuelle, dans une perspective externe, consiste à se représenter la réalisation d'un mouvement ou d'une séquence de mouvements du point de vue du spectateur. Dans une perspective interne, l'imagerie visuelle consiste à s'imaginer les changements associés à la réalisation du mouvement du point de vue de l'exécutant. Enfin, l'imagerie proprioceptive consiste à évoquer mentalement les sensations produites par l'exécution d'un mouvement. Bien que peu nombreux, les travaux contrastant ces différents types d'imagerie soulignent la nécessité de prendre en compte les contraintes de la tâche à réaliser dans l'évaluation des différentes modalités d'imagerie (Hall et al., 1992). Comparée à une perspective interne, l'imagerie visuelle externe apparaît bénéfique pour assurer la précision d'actions motrices pour lesquelles le succès repose sur l'exactitude d'une forme à produire, comme c'est le cas par exemple lors de la réalisation d'un kata, d'une séquence de gymnastique ou d'une forme graphique (Denis et Boucher, 1991 ; Féry, 2003 ; Hardy et Callow, 1999 ; White et Hardy, 1995). En revanche, une perspective visuelle interne semble plus appropriée pour des tâches au cours desquelles les contraintes de planification d'une action en réponse aux changements intervenant dans le champ visuel sont fortes (exemple du slalom en kayak ; Hardy et Callow, 1999 ; White et Hardy, 1995). L'imagerie proprioceptive favorise, quant à elle, l'apprentissage des aspects temporels des mouvements ou de la coordination inter-segmentaire (Féry, 2003 ; Féry et Morizot, 2001) et conduit à une amélioration maximale de la précision des formes lorsqu'elle est utilisée conjointement à l'imagerie visuelle externe (Hardy et Callow, 1999). Bien que ces études soulignent l'importance de guider les sujets vers une pratique d'imagerie visuelle externe dans des tâches de reproduction de formes, ou vers une pratique mixte (vision + proprioception), aucune étude n'a inclus une comparaison directe entre des instructions sollicitant soit l'imagerie visuelle, soit l'imagerie proprioceptive, pour déterminer leur importance respective dans l'apprentissage moteur. 
Dans le cadre de ce travail, l'importance des modalités d'imagerie visuelle (dans une perspective externe) et proprioceptive sollicitées lors d'une pratique mentale est questionnée dans une tâche de positionnement des segments corporels des membres inférieurs. Cette tâche consiste, en l'absence d'indices visuels, à placer correctement la jambe gauche dans une position déterminée au préalable. Les travaux dédiés à l'étude des facteurs intervenant sur la précision de la reproduction d'une position angulaire reconnaissent qu'un placement correct des membres inférieurs reposant sur le traitement exclusif des indices proprioceptifs peut être reproduit dans une situation sollicitant la modalité visuelle (Cadopi, 1980 ; Euzet et Gahéry, 1995, 1996 ; Rodier, Euzet, Gahéry et Paillard, 1991). Les résultats obtenus montrent que les individus parviennent sans trop de difficultés à faire correspondre, en actionnant une manette, le schéma d'une position angulaire du genou présenté sur un écran à la position angulaire de leur jambe dissimulée sous une table, ou à adopter une position angulaire de la jambe présentée à l'écran en mobilisant activement l'une de leurs jambes. Les possibilités de transfert intermodal mises en évidence dans ces études, ainsi que les données rapportées dans la littérature sur les effets de l'imagerie, conduisent à supposer qu'une pratique sollicitant l'imagerie visuelle externe devrait être bénéfique au développement de la précision angulaire des formes corporelles à reproduire. Bien qu'un effet bénéfique de l'imagerie proprioceptive soit également attendu après plusieurs essais de pratique, l'imagerie proprioceptive nécessitant une certaine expertise pour s'exprimer (Hardy et Callow, 1999), aucune étude à ce jour concernant la répétition mentale d'une action ne permet de prédire laquelle de ces deux pratiques (imagerie visuelle versus imagerie proprioceptive) conduira à une amélioration maximale des capacités à reproduire le plus précisément possible des formes corporelles simples. On pourrait cependant s'attendre à ce que, dans une tâche où seules les informations proprioceptives sont sollicitées, l'imagerie proprioceptive apporte davantage de bénéfices que l'imagerie visuelle sur la précision du placement de la jambe. Les résultats des travaux comparant la précision de l'évaluation du positionnement de segments corporels en situation intramodale (lorsque référence et reproduction font intervenir la modalité proprioceptive uniquement) et intermodale (lorsque référence et reproduction font intervenir deux modalités sensorielles différentes : vision et proprioception) apporteraient des arguments en faveur de cette hypothèse. En effet, une plus grande précision de la performance motrice apparaît lorsque seule la modalité proprioceptive est sollicitée (Cadopi, 1980, 
1992 ; Euzet et Gahéry, 1995, 1996 ; Rodier et al., 1991). L'étude présentée ici consiste donc en une approche comparative des performances motrices découlant d'une pratique par imagerie visuelle ou proprioceptive.

\section{Matériel et méthode}

\section{Sujets}

Vingt sujets droitiers (12 femmes et 8 hommes), âgés de 23,5 ans $( \pm 2,7$ ans), sans spécialité sportive particulière, ont participé volontairement à cette expérience. Ils n'ont été informés du but de cette étude qu'une fois la passation terminée. Aucun d'entre eux ne présentait de trouble ou d'antécédent particulier ayant trait à leur vision et à leur motricité.

\section{Procédure expérimentale}

L'expérience s'est déroulée en 4 phases réparties sur 2 jours. La première phase (pré-test) consistait à évaluer la précision de la reproduction de positions angulaires sollicitant l'articulation du genou en dehors de toute possibilité de contrôle visuel (yeux fermés). Les sujets étaient assis confortablement sur une table surélevée et pouvaient réaliser aisément des extensions de la jambe gauche à partir de la position repos (jambe pendant dans le vide). Après avoir équipé les sujets d'un potentiomètre goniométrique placé sur l'articulation du genou gauche et fixé au mollet et à la cuisse par des velcros, l'expérimentateur mesurait la position de repos des sujets assis sur la table. Cette valeur angulaire servait de position de référence pour les phases ultérieures de l'expérience. Les sujets déplaçaient ensuite activement leur jambe jusqu'à atteindre une des trois positions angulaires retenues pour cette étude (position d'encodage: $132^{\circ}, 147^{\circ}$ et $162^{\circ}$ ), et définies chacune par une butée. Ces positions s'échelonnent entre $90^{\circ}$, valeur correspondant à la position repos, et $180^{\circ}$, valeur correspondant à l'extension maximale de la jambe. Les sujets maintenaient la position pendant 4 secondes, avant de ramener leur jambe en position de repos. Moins d'une seconde plus tard, ils devaient reproduire (position de rappel) le plus précisément possible la position préalablement encodée. Les sujets n'étaient pas informés de l'exactitude de leur réponse. Douze essais présentés aléatoirement (4 essais pour chacune des 3 configurations angulaires imposées) étaient réalisés par 
chaque sujet. Pour s'assurer de la compréhension des consignes et du déroulement d'un essai (phases successives d'encodage et de rappel), deux essais blancs correspondant aux angles de $139^{\circ}$ et $154^{\circ}$ ont été utilisés.

Lors de la deuxième phase expérimentale (phase de pratique mentale), les sujets ont été séparés aléatoirement en deux groupes. Le groupe IV participait à une pratique par Imagerie Visuelle (selon une perspective externe), tandis que le groupe IP participait à une pratique par Imagerie Proprioceptive. Les sujets du groupe IV avaient pour consigne de s'imaginer se voir placer leur jambe gauche dans la position précisée par l'expérimentateur, tandis que les sujets du groupe IP avaient pour consigne d'évoquer mentalement les sensations accompagnant l'exécution du placement de leur jambe. Quel que soit le type de pratique (IV versus IP), la réalisation d'un essai d'imagerie s'effectuait sur une durée d'environ 8 secondes ${ }^{(2)}$. Avant que ne débute cette phase expérimentale, les 3 positions à réaliser étaient rappelées aux sujets. Les positions utilisées au cours des pratiques en imagerie étaient identiques à celles utilisées lors des pré-tests $\left(132^{\circ}, 147^{\circ}\right.$ et $\left.162^{\circ}\right)$ et leur présentation était aléatoire. Dans chacun des groupes, les sujets devaient réaliser 3 séries de 30 essais sans faire aucun mouvement, leurs jambes restant en position de repos.

Les phases 3 et 4 (post-tests), réalisées respectivement 10 minutes et 24 heures après la fin de la phase de pratique mentale, étaient en tout point identiques au pré-test.

\section{Analyse des données}

Pour un essai donné, le potentiomètre permettait de mesurer l'écart entre les positions d'encodage et les positions de rappel lors du pré-test et des post-tests. Cet écart correspondait à l'erreur de positionnement. La moyenne des erreurs absolues, calculée pour chaque sujet, a été retenue comme variable dépendante ${ }^{(3)}$, ainsi que l'erreur variable. Les erreurs de positionnement étant identiques pour chacune des 3 positions utilisées

(2) Durée correspondant à la pratique réelle de la tâche : placement de la jambe dans une position spécifique, maintien de la jambe dans cette position pendant 4 secondes et retour en position de repos.

(3) Une analyse signée des erreurs a été menée (CE). L'ensemble des erreurs se traduisait par une surestimation des positions de rappel, quels que soient le groupe, la phase expérimentale et la position. Aucun effet significatif lié à l'un de ces facteurs ou à leur interaction n'a été observé. 
dans cette expérience $\left(1.94^{\circ}\right.$ en moyenne $\left.\pm 0.13^{\circ}\right)$, les analyses statistiques ont été réalisées à partir des erreurs de positionnement moyennes, toutes positions confondues.

Des analyses de variances ont été menées sur l'erreur absolue et l'erreur variable avec le Groupe (pratique IV versus pratique IP) comme facteur inter-sujets et la Phase Expérimentale (pré-test, post-test 10', post-test $24 \mathrm{~h}$ ) comme facteur intra-sujets. Les comparaisons de moyennes ont été réalisées au moyen du test de Newman-Keuls. Les résultats ont été considérés comme significatifs pour un niveau de probabilité inférieur à 0.05 .

\section{Résultats}

L'analyse de l'erreur absolue ne révèle pas d'effet principal du Groupe, $\mathrm{F}(1,18)=3.85, \mathrm{p}=0.07$, et de la Phase Expérimentale, $\mathrm{F}(2,36)=0.20$, $\mathrm{p}=0.82$, tandis $\mathrm{qu}^{\prime}$ une interaction significative entre ces deux facteurs apparaît, $\mathrm{F}(2,36)=16.85, \mathrm{p}<0.001$. Comme illustré sur la figure 1 , l'erreur absolue du groupe IP diminue du pré-test aux post-tests 10' et 24 heures, sans qu'aucune différence n'apparaisse entre les deux posttests. En revanche, l'erreur absolue du groupe IV augmente du pré-test au post-test $10^{\prime}$, pour revenir à son niveau initial 24 heures après la fin de la phase de pratique mentale.

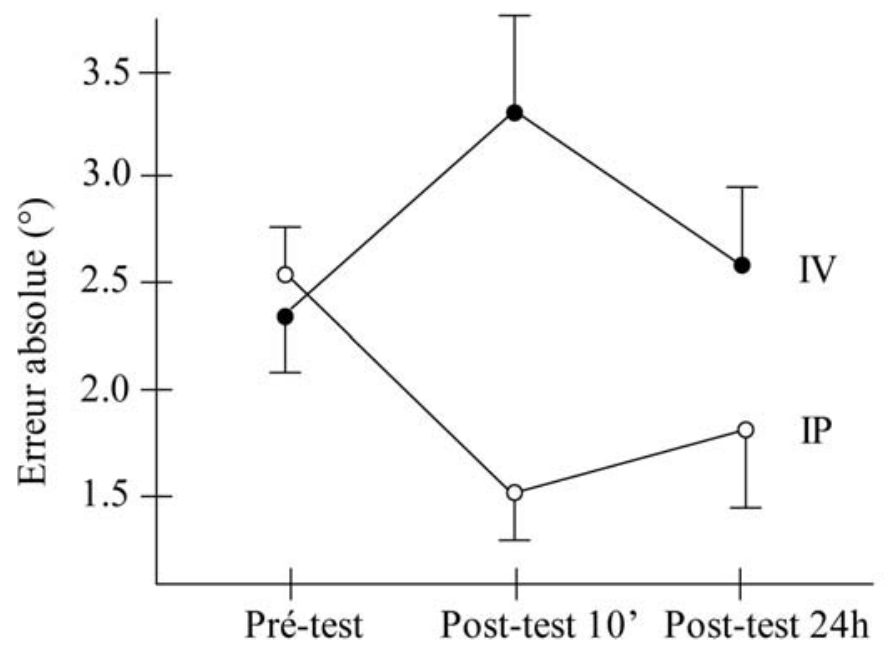

FIGURE I

Erreur absolue (en degré) en fonction de la modalité d'imagerie (Imagerie visuelle ou IV versus Imagerie Proprioceptive ou IP) et de la phase expérimentale (pré-test, post-test 10 minutes, post-test 24 heures) 
L'analyse de l'erreur variable révèle un effet principal du Groupe, $\mathrm{F}(1,18)=13.67, \mathrm{p}<0.01$, et de la Phase Expérimentale, $\mathrm{F}(2,36)=4.13$, $\mathrm{p}<.05$, ainsi qu'une interaction significative entre ces deux facteurs, $\mathrm{F}(2,36)=7.33, \mathrm{p}<0.01$. Cette interaction résulte d'une diminution significative de l'erreur variable aux post-tests (10' et $24 \mathrm{~h}$ ) dans le groupe IP, alors qu'aucun changement n'apparaît dans le groupe IV (figure 2).

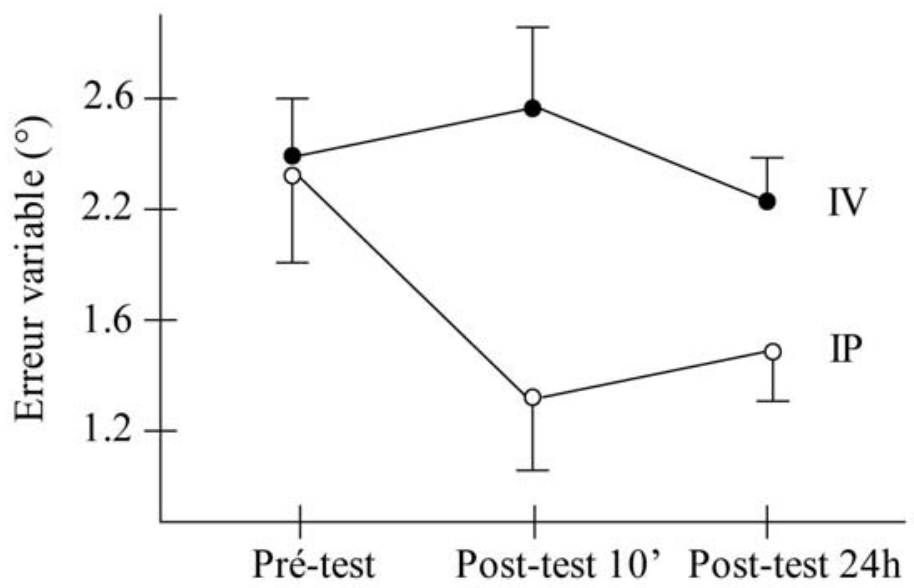

FIGURE 2

Erreur variable (en degré) en fonction de la modalité d'imagerie (Imagerie visuelle ou IV versus Imagerie Proprioceptive ou IP) et de la phase expérimentale (pré-test, post-test 10 minutes, post-test 24 heures)

\section{Discussion}

Ce travail avait pour but de déterminer l'importance respective des pratiques d'imagerie visuelle et proprioceptive sur l'amélioration de la reproduction de configurations corporelles simples, évaluée dans une tâche de placement " en aveugle » de la jambe dans une position déterminée. Les principaux résultats obtenus dans cette expérience rapportent que seule une pratique par imagerie proprioceptive est bénéfique au développement de la précision de la reproduction de formes corporelles. Contrairement à nos attentes, une pratique par imagerie visuelle (selon une perspective externe) se traduit par une détérioration temporaire de la performance motrice (post-test 10 minutes), pour revenir à son niveau de base après 24 heures. Selon Hardy (1997), la supériorité d'une pratique par imagerie visuelle externe dans l'apprentissage et le contrôle d'une tâche reposant sur la forme produite s'expliquerait par l'apport d'infor- 
mations supplémentaires à celles normalement disponibles. L'imagerie visuelle externe permet notamment à l'exécutant de se représenter mentalement et avec précision les différentes positions et mouvements nécessaires au succès de la performance. Dans la tâche utilisée dans cette étude, la simplicité des formes corporelles produites, sollicitant uniquement l'articulation du genou et n'impliquant pas l'enchaînement d'une séquence d'actions, contrairement à la réalisation d'une routine en gymnastique par exemple, pourrait expliquer l'absence d'effet bénéfique découlant d'une pratique par imagerie visuelle. Contrairement à la modalité proprioceptive, la modalité visuelle n'apporterait pas d'indices vraiment utiles dans une tâche de positionnement de segments corporels effectuée sans l'aide de la vision.

Les travaux dédiés au développement du sens de la position confirment l'importance de la modalité proprioceptive dans la reproduction d'une position angulaire des membres supérieurs ou inférieurs (Cadopi, 1980, 1992 ; Euzet et Gahéry, 1995, 1996 ; Rodier et al., 1991). Dans ces travaux, la précision de la performance motrice est effectivement supérieure en situation intramodale, faisant intervenir la modalité proprioceptive uniquement, qu'en situation intermodale, dans laquelle un transfert d'information est réalisé entre modalités visuelle et proprioceptive. Dans le cadre de notre expérience, le préjudice lié au transfert $\mathrm{d}$ 'information entre systèmes visuel et proprioceptif s'exprimerait par un apprentissage inefficace (post-test 24 heures), voire néfaste (post-test 10 minutes) à la précision du sens de la position lorsque la répétition mentale de l'action sollicitait la modalité visuelle. Ces résultats suggèrent que la répétition de l'imagerie visuelle conduit les individus à délaisser temporairement les informations proprioceptives, expliquant la dégradation de la performance motrice au post-test réalisé quelques minutes après la séance de pratique par imagerie. Cette interprétation suppose que la pratique mentale visuelle aurait des effets similaires à une pratique physique visuellement guidée. En effet, dans ce dernier cas, certains travaux ont montré que la sollicitation du système visuel lors d'une séance d'apprentissage par pratique physique pouvait entraver le traitement des indices en provenance d'autres systèmes sensoriels (Adams, Gopher et Lintern, 1977 ; Robin, Proteau, Toussaint \& Blandin, en révision), cet effet s'amenuisant peu à peu lorsque les indices proprioceptifs jouent un rôle déterminant pour le succès de la tâche à réaliser (Robin, Toussaint, Blandin et Vinter, sous presse). D'une façon générale, il ressort de ces travaux que l'importance des modalités sensorielles pour l'atteinte d'une précision motrice optimale varie en fonction 
des contraintes de la tâche, notamment de la place prépondérante accordée soit aux rétroactions d'origine visuelle (Proteau et Carnahan, 2001 ; Tremblay et Proteau, 1998), soit aux rétroactions d'origine proprioceptive (Robin et al., sous presse ; Tremblay, Welsch et Elliott, 2001). Les résultats obtenus dans ce présent travail apportent des arguments en faveur de l'effet bénéfique d'une similitude entre les modalités d'imagerie et les modalités sensorielles impliquées dans la tâche à réaliser lors d'un apprentissage par imagerie mentale. Cette conclusion, découlant d'une tâche dans laquelle la modalité sensorielle dominante est de nature proprioceptive, reste toutefois à confirmer dans des tâches à dominante visuelle. Des travaux sont actuellement en cours dans notre laboratoire pour examiner l'importance des contraintes de la tâche et de la spécificité des conditions d'apprentissage lors d'une pratique par imagerie.

Conclure à la dominance d'une pratique par imagerie proprioceptive sur l'amélioration de la reproduction précise de formes corporelles simples nécessitait de notre part un contrôle des différences inter-individuelles en matière de capacités d'imagerie (Goss, Hall, Buckolz et Fishburne, 1986 ; Hall et al., 1992). La mauvaise performance du groupe IV pouvait effectivement résulter de difficultés éprouvées par les sujets à former des images visuelles des actions à réaliser, alors que de telles difficultés d'imagerie ne seraient pas rapportées dans le groupe IP. Les capacités $\mathrm{d}$ 'imagerie visuelle et proprioceptive des sujets des groupes IV et IP respectivement ont été évaluées, quelques jours après l'expérience, au moyen du Questionnaire en Imagerie du Mouvement (ou MIQ) de Hall et Pongrac (1983). Dans ce test, plus un score est élevé, plus il traduit des difficultés à former des images mentales à partir de la modalité sensorielle sollicitée (imagerie visuelle versus imagerie proprioceptive). Les scores moyens obtenus par le groupe IV sont de $13.35( \pm 2.74)$ et $23.5( \pm 5.77)$ pour l'imagerie visuelle et proprioceptive respectivement, et de 14.5 $( \pm 3.66)$ et $21.35( \pm 3.67)$ dans le groupe IP. Ces scores indiquent de plus grandes facilités d'imagerie dans le groupe IV que dans le groupe IP pour la modalité sensorielle sollicitée dans les phases de pratique par imagerie (visuelle et proprioceptive respectivement). Par conséquent, la supériorité d'une pratique sollicitant l'imagerie proprioceptive sur le développement de la précision du sens de la position n'est pas remise en cause par l'évaluation des capacités moyennes d'imagerie de chacun des groupes. 


\section{Bibliographie}

ADAMS, J.A., GOPHER, D., \& LINTERN, G. (1977). Effects of visual and proprioceptive feedback on motor learning. Journal of Motor Behavior, 9, 11-22.

CADOPI, M. (1980). L'élaboration de la référence interne dans une morphocinèse : relations entre les représentations visuelles et les représentations kinesthésiques. Cahiers de Psychologie, 23, 87-95.

CADOPI, M. (1992). Représentation de l'orientation du corps et de la vitesse du mouvement en danse chez les enfants de 10 ans. STAPS, 9, 19-31.

DECETY, J., \& JEANNEROD, M. (1996). Mentally simulated movements in virtual reality: Does Fitt's law hold in motor imagery? Behavioural Brain Research, 72, 127-134.

DeCETY, J., JeAnNERod, M., DurozARD, D., \& BAVERel, G. (1993). Central activation of autonomic effectors during mental simulation of motor actions. Journal of Physiology, 461, 549-563.

DeCETY, J., JEANNEROD, M., GERMAIn, M., \& PASTENE, J. (1991). Vegetative response during imagined movement is proportional to mental effort. Behavioural Brain Research, 42, 1-5.

DeCETY, J., JEANNEROD, M., \& PRABlANC, C. (1989). The timing of mentally represented actions. Behavioural Brain Research, 34, 35-42.

DECETY, J., \& MiCHEL, F. (1989). Comparative analysis of actual and mental movement times in two graphics tasks. Brain and Cognition, 11, 87-97.

DENIS, S., \& BOUCHER, J.L. (1991). La représentation spatiale d'un tracé bi-dimensionnel. Revue Canadienne de Psychologie, 45, 405-414.

EUZET, J.-P., \& GAHÉRY, Y. (1995). Relationships between position sense and physical practice. Journal of Human Movement Study, 28, 149-173.

EUZET, J.-P., \& GAHÉRY, Y. (1996). Kinesthésie et motricité: Influence de la pratique sur le sens de la position. Science et Motricité, 29-30, 30-40.

FELTZ, D.L., \& LANDERS, D.M. (1983). The effects of mental practice on motor skill learning and performance: A meta-analysis. Journal of Sports Psychology, 5, 25-57.

FÉRY, Y.A. (2003). Differentiating visual and kinesthetic imagery in mental practice. Canadian Journal of Experimental Psychology, 57, 1-10.

FÉRY, Y.A., \& MORIZOT, P. (2001). Kinesthetic and visual images in modelling closed motor skills : the example of the tennis serve. Perceptual and Motor Skills, 90, 707-722.

Goss, S., HALl, C.R., BuCKOLZ, E., \& FiSHBURNE, G.J. (1986). Imagery ability and the acquisition and retention of movements. Memory and Cognition, 14, 469-477.

HALL, C.R., BuCKOlZ, E., \& FishbURNE, G.J. (1992). Imagery and the acquisition of motor skills. Canadian Journal of Sport Science, 17, 19-27.

HALl, C.R., \& PONGRAC, J. (1983). Movement Imagery Questionnaire. London, Ontario: University of Western Ontario.

HARDY, L. (1997). Three myths about applied consultancy work. Journal of Applied Sport Psychology, 9, 277-294. 
HARDY, L., \& CALLOW, N. (1999). Efficacy of external and internal visual imagery perspectives for the enhancement of performance on tasks in which form is important. Journal of Sport and Exercise Psychology, 21, 95-112.

INGVAR, D., \& PHILIPSSON, L. (1977). Distribution of the cerebral blood flow in the dominant hemisphere during motor ideation and motor performance. Annals of Neurology, 2, 230-237.

JEANNEROD, M. (1999). Perspectives on the Representation of Actions. The Quarterly Journal of Experimental Psychology, 52A, 1-29.

PROTEAU, L., \& CARNAHAN, H. (2001). What causes specificity of practice in a manual aiming movement: vision dominance or transformation errors? Journal of Motor Behavior, 33, 226-234.

Robin, C., Proteau, L., ToussainT, L., \& BlandiN, Y. (en révision). Specificity of learning in a video-aiming task: modifying the salience of dynamic visual cues. Journal of Motor Behavior.

RoBin, C., TOUSSAINT, L., BlANDIN, Y., \& VINTER, A. (in press). Sensory integration in the learning of aiming towards "self-defined" targets. Research Quarterly for Exercise and Sport.

RODIER, S., EUZET, J.P., GAHÉRY, Y. \& PAILlARD, J. (1991). Cross-modal versus intramodal evaluation of the knee joint angle. A normative study in a population of young adults. Human Movement Science, 10, 689-712.

Roure, R., Collet, C., Deschaumes-Molinaro, C., Delhomme, G., DitTmar, A. \& VERNET-MAURY, E. (1999). Imagery quality estimated by autonomic response is correlated to sporting performance enhancement. Physiology and Behavior, $66,63-72$.

Sirigu, A., Duhamel, J.R., Cohen, L., Pillon, B., Dubois, B. \& AGID, Y. (1996). The mental representation of hand movements after parietal cortex damage. Science, 273, 1564-1568.

TREMblay, L. \& Proteau, L. (1998). Specificity of practice: The case of powerlifting. Research Quarterly for Exercise and Sport, 69, 284-289.

TREMblay, L., Welsh, T. N. \& ElLIOTT, D. (2001). Specificity versus variability: Effects of practice conditions on the use of afferent information for manual aiming. Motor Control, 5, 347-360.

WHITE, A. \& HARDY, L. (1995). Use of different imagery perspectives on the learning and performance of different motor skills. British Journal of Psychology, 86, 169-180. 